Sādhanā Vol. 40, Part 8, December 2015, pp. 2367-2379. (C) Indian Academy of Sciences

(D) CrossMark

\title{
Thrust generation and wake structure for flow across a pitching airfoil at low Reynolds number
}

\author{
INTESAAF ASHRAF, AMIT AGRAWAL*, \\ MAJID HASSAN KHAN, SOORAJ P, ATUL SRIVASTAVA \\ and ATUL SHARMA
}

Department of Mechanical Engineering, Indian Institute of Technology Bombay, Powai, Mumbai 400076, India

e-mail: amit.agrawal@iitb.ac.in

MS received 20 September 2014; revised 19 May 2015; accepted 3 August 2015

\begin{abstract}
In this work, we present detailed particle image velocimetry (PIV) based investigation of wake structure of a pitching airfoil. PIV measurements have been carried out for NACA0015 airfoil at $\mathrm{Re}=2900$ with reduced frequency range of 1.82-10.92 and pitching angle of $5^{\circ}$. Two different wake structures (reverse Kármán shedding and deflected vortex shedding) are observed over this parameter range. The vorticity decreases substantially over a distance of two chord-lengths. The velocity profile indicates a jet-like flow downstream of the airfoil. It is shown that the jet-like flow downstream of the airfoil is however not a sufficient condition for the generation of thrust. The vortex strength is found to be invariant of the pitching frequency. Certain differences from the reported results are noted, which may be because of difference in the airfoil shape. These results can help improve understanding of the flow behavior as the low Reynolds number range is not well studied.
\end{abstract}

Keywords. Pitching airfoil; thrust generation; particle image velocimetry.

\section{Introduction}

Birds, insects and fish generate lift and thrust by using heaving and pitching motions of their wings or fins (Govardhan \& Arakeri 2011). The dynamics of pitching airfoil therefore forms a subject of great practical and theoretical importance. In 1490, Leonardo da Vinci tried to explain and implement the mechanism of thrust generation by a flapping wing. Since then numerous attempts have been made to develop flight vehicles using flapping wings, especially at the end of the 19th century and beginning of 20th century. Recent advances in technology have opened the possibility of building small machines (such as Micro/Nano Air Vehicles) from inspiration of these living creatures (Shyy et al 2010). Research interest is growing on these vehicles because

*For correspondence 
of their unique characteristics, such as small size, high maneuverability, and great hovering capabilities.

The generation of thrust by a flapping airfoil depends upon the wake structure behind the trailing edge of the airfoil. When a stationary airfoil or low frequency flapping airfoil is placed in a flow, the wake behind an airfoil is a Kármán vortex street; consisting of a row of clockwise vortices above the mean line and a row of anticlockwise vortices below this mean line. These contra-rotating vortices give rise to a drag producing vortex wake. As the oscillation frequency increases, the wake structure behind the airfoil changes to a reverse Kármán vortex street. Such a wake vortex pattern causes generation of net thrust on the airfoil. This wake structure is also known as a "jet" and is a result of momentum excess behind the airfoil. The thrust producing phenomenon due to flapping airfoil in a fluid flow is also known as the Knoller-Betz or the Katzmyer effect (Jones et al 1998).

Koochesfahani (1989) experimentally observed that thrust generation by pure pitching of an airfoil is possible. He calculated the propulsive force on the airfoil by using the integral momentum equation. He reported that thrust generation occurs above a critical frequency; the critical frequency decreases as the pitch amplitude increases. Bohl \& Koochesfahani (2009) presented molecular tagging velocimetry based velocity and vorticity measurements for NACA0012 airfoil, at Reynolds number (based on chord) of 12,600 and reduced frequency range of 4.111.5. They employed the wake survey method, with appropriate correction for pressure forces, and found that their results match well with the simulation results of Ramamurti \& Sandberg (2001). The transverse alignment of the vortices changes beyond a reduced frequency of 5.7; however, this did not correspond to a crossover from drag to thrust. Schnipper et al (2009) performed flow visualization in a soap tunnel using an airfoil with thickness $1 / 6^{\text {th }}$ of the chord, at Reynolds number (based on thickness) of 220 and 440. They performed measurements at several frequencies and amplitudes and presented evidence for occurrence of several different flow regimes including $2 \mathrm{~S}$ and $2 \mathrm{P}$. They argued that transition from Kármán wake to inverted Kármán wake occurs approximately at Strouhal number (based on amplitude) of 0.18. Freymuth (1988) experimentally observed thrust generating wake patterns in pure pitch and pure heave conditions. He also discussed the effect of changing pitch and plunge amplitude, as well as the mean angle of attack. Sarkar \& Venkatraman (2006) performed numerical simulations using the discrete vortex method for flow past a pitching airfoil at $\operatorname{Re} \sim 10,000$. They studied the effect of varying oscillation frequency, amplitude of oscillation, mean angle of attack, and location of the pitching axis on the vortex structure and drag coefficient. Drag producing wake was noticed at small frequencies which changed to thrust producing wake at higher frequencies. The results were found to be in qualitative agreement with the inviscid theory predictions; however, substantial differences were noted at larger mean angle of attack. Chandar \& Damodaran (2010) presented three-dimensional simulations of a pitching airfoil. Shinde \& Arakeri (2013) performed measurements with an airfoil pitching in otherwise stationary water. The flow was studied at three amplitudes and four frequencies, with Reynolds number (based on the maximum trailing edge velocity and chord length) of 1078-8625. Cleaver et al (2012) present particle image velocimetry (PIV) based measurements at Re $=10,000$ on NACA0012 airfoil. Buchholz \& Smits (2008) and Green \& Smits (2008) studied pitching of a rectangular panel. See Platzer et al (2008) for a review on aerodynamics of flapping airfoil. More recently Yu et al (2013a, b) carried about numerical investigation of pure plunging, pure pitching and combined pitching and plunging motions. They studied the effect of kinematics and thickness on propulsive performance and vortex dynamics. In case of pure plunging motion, thicker airfoil was found to generate higher thrust as compared to thin airfoil. But for combined motion, thin airfoil outperforms thick airfoil by a factor of almost 4 . They attributed this to high frontal area and dynamics 
of leading edge vortices. They observed that at low Strouhal number $(=0.3)$, thin airfoils are superior and independent of the reduced frequency. However at relatively high Strouhal number $(=0.45)$, different airfoils are dependent on the reduced frequency.

The literature survey suggests that most of the existing studies are with NACA0012 airfoil and Reynolds number greater than 10,000. The present study with NACA0015 airfoil and $\operatorname{Re}=2900$ covers a somewhat different parameter range. Such low Reynolds number experimental data will be particularly useful for benchmarking numerical simulations. We note that quantitative vorticity measurements for flow around pitching airfoil are not readily available; these issues provided the motivation for undertaking this work.

\section{Experimental setup}

The experiments have been carried out in a low Reynolds number water channel. Figure 1a shows the schematic diagram of the experimental facility. The size of the water channel is 1.40 $\times 0.40 \times 0.40 \mathrm{~m}^{3}$. Water is pumped into the channel using a 6 liter per second capacity pump. Two-dimensional PIV technique is used for wholefield velocity measurements. The PIV system consists of (Sewatkar et al 2012) an Nd:YAG double-pulsed laser (wavelength $532 \mathrm{~nm}$, energy $200 \mathrm{~mJ}$ per pulse, repetition rate $15 \mathrm{~Hz}$; from Beamtech, China), tracer particles (spherical hollow fused borosilicate glass particles; mean particle size of $8-11 \mu \mathrm{m}$, specific density around 1.1; from Potter Industries Inc., Australia), an inter-line CCD camera (PCO, Germany; image size $1392 \times 1024$ pixels, frame rate $10 \mathrm{~Hz}$ ), a synchronizer (from Beamtech) and a beam-arm. The beam coming out from the laser undergoes multiple reflections inside the beam arm, and finally gets converted into a light sheet after passing through a pair of cylindrical and spherical lenses. The beam-arm allows for a quick and easy positioning of the light sheet. The time delay between a pair of PIV images is suitably adjusted and varied with the flapping frequency. The images obtained from the camera are processed using a commercial code to obtain velocity vectors and vorticity contours. The analysis of images was carried out for interrogation spot size of $32 \times 32$ pixels, with $50 \%$ overlap, yielding about 5418 vectors. The vectors are therefore 16 pixels (or about $1.2 \mathrm{~mm}$ ) apart. The percentage of bad vectors in a PIV frame was less than $1 \%$. The typical error in velocity measurement is estimated as $1.3 \%$.

The experiments were carried out for NACA0015 airfoil having chord length $(c)$ of $5 \mathrm{~cm}$ and span of $32 \mathrm{~cm}$. The material of the airfoil is acrylic and the airfoil was manufactured using CNC machining. Care was taken to ensure that the shape and dimension of the airfoil are as per the specifications. The free stream velocity $\left(U_{o}\right)$ was $0.043 \mathrm{~m} / \mathrm{s}$ and the corresponding Reynolds number (calculated from $c U_{o} / v$ where $v$ is the kinematic viscosity of water) is 2900 . The direction of illumination was carefully chosen such that the shadow region in the view frame was kept to the minimum possible. The view frame encompassed the trailing edge of the airfoil and extended up to 2.2 chord lengths downstream of the airfoil, and had a width of 1.7 times the chord.

The pitching of airfoil takes place at the leading edge and is sinusoidal in nature. The pitching mechanism consists of a steel rod connecting a stepper motor to the airfoil, as shown in figure 1c. The holding torque of the motor is $0.87 \mathrm{~kg}-\mathrm{m}$. The pitching frequency and amplitude of the airfoil can be programmed through a PC, which suitably controls the stepper-motor. Microstepping is used in programming of the motor with a minimum step size of $0.18^{\circ}$. It was verified that the pitching profile is sinusoidal.

The airfoil was flapped at six different frequencies $(f=0.5,1.0,1.5,2.0,2.5$ and $3.0 \mathrm{~Hz})$ at a constant flapping angle $\left(\alpha= \pm 5^{\circ}\right.$ or non-dimensional amplitude defined as twice the amplitude 


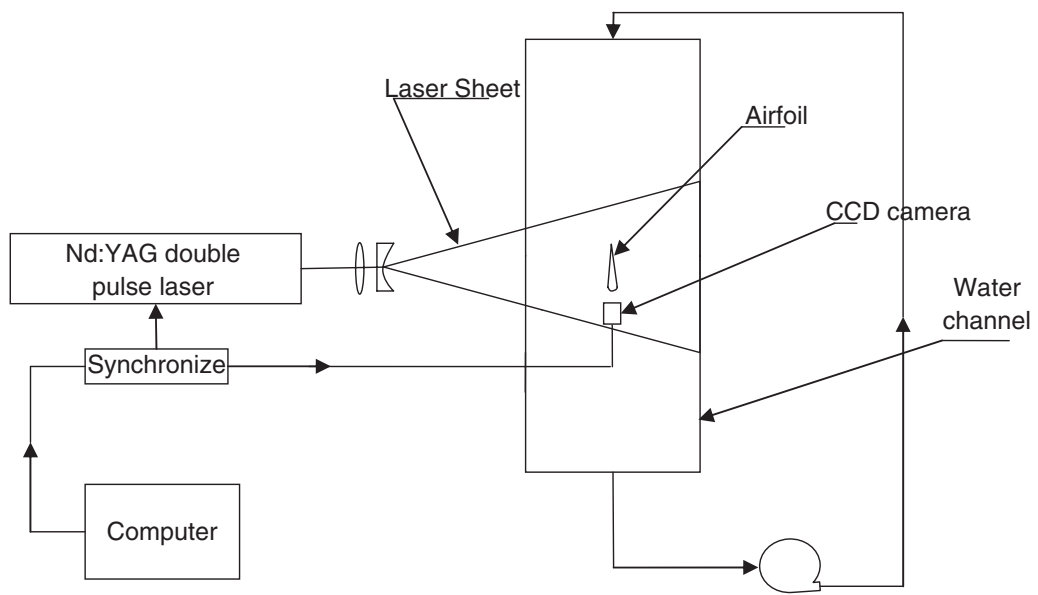

(a)

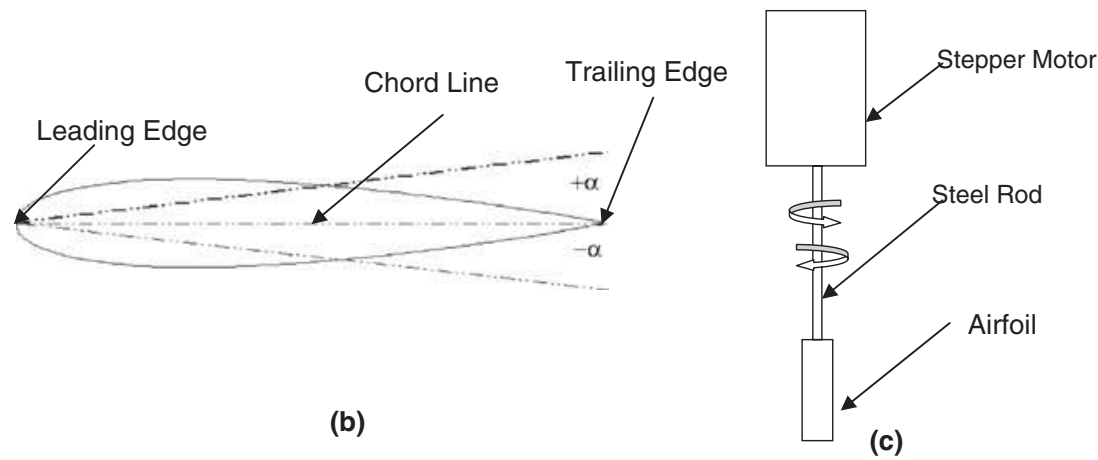

Figure 1. (a) Schematic of the experimental setup employed in the experiments, (b) schematic of the pitching airfoil, (c) schematic of the arrangement employed for pitching the airfoil.

divided by chord of 0.175 ). The corresponding reduced frequencies (defined as $k=\pi f c / U_{o}$ ) are 1.82-10.92, in step of 1.82. The reduced frequency is a measure of the relative importance of acceleration reaction to pressure drag and lift forces, and measures flow unsteadiness imposed by flapping motion. The reduced frequency is typically employed for expressing the frequency in non-dimensional form. Note that pitching occurs about zero mean angle of attack.

\section{Results}

The flow structures as revealed by the velocity vectors and vorticity contours are investigated as a function of flapping frequency in this section. The variation in velocity profile and vortex size and strength as a function of flapping frequency is also examined.

\subsection{Instantaneous wake structure}

Figure 2 presents the instantaneous vorticity $(\omega)$ contours, velocity vectors, velocity contours, and size and strength of vortices for reduced flapping frequency of 1.82 respectively, for three time instants. The origin of the coordinate system is fixed at the tip of the airfoil, at its 

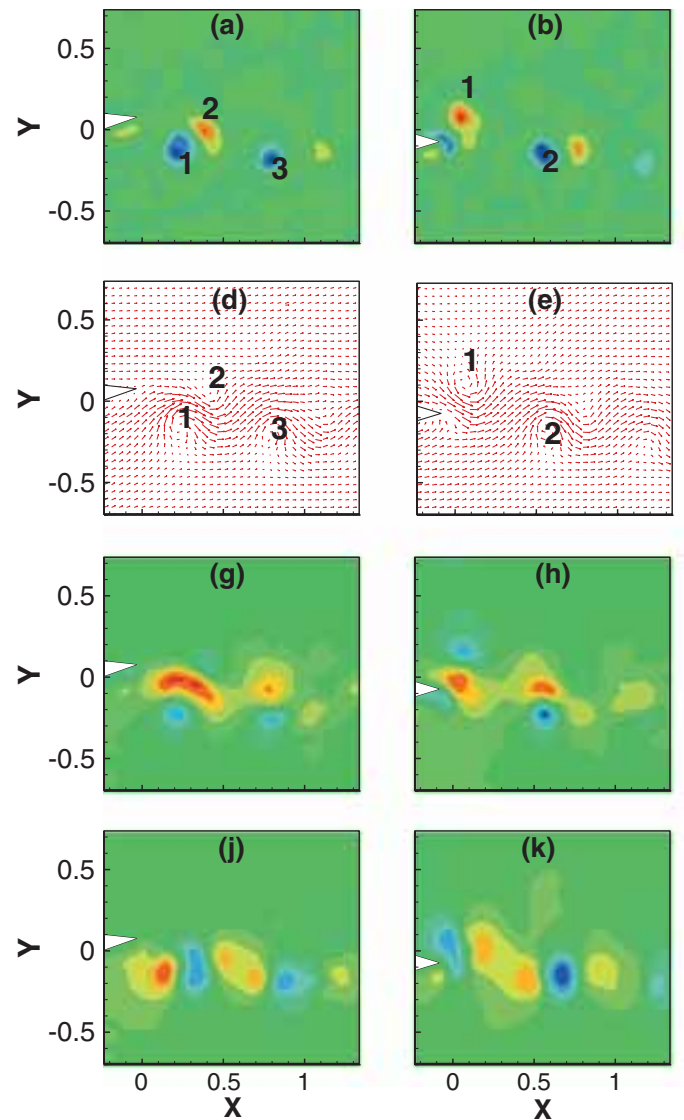

(m)

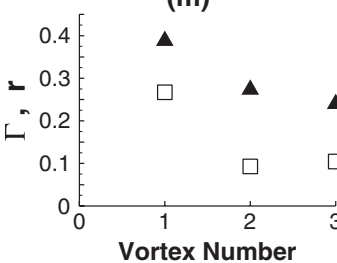

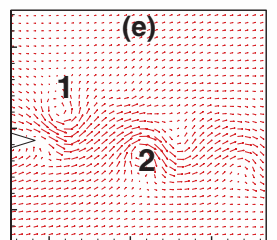
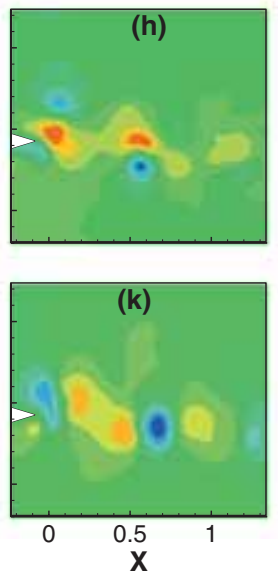

(n)

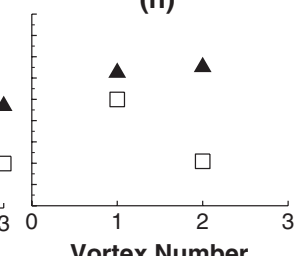

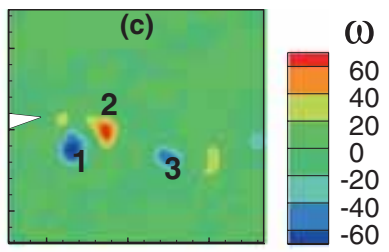
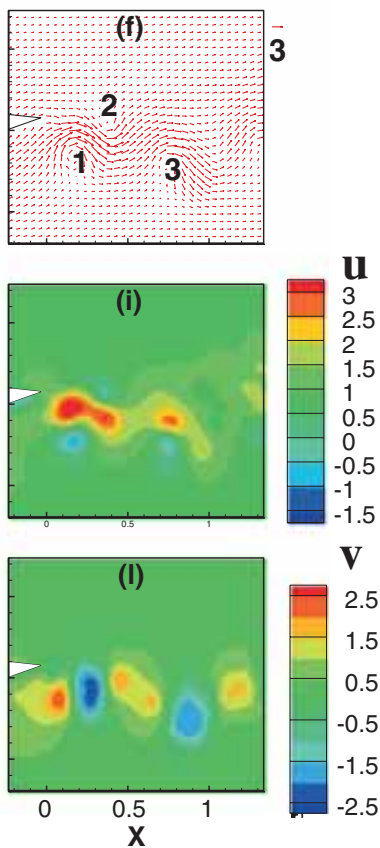

(o)

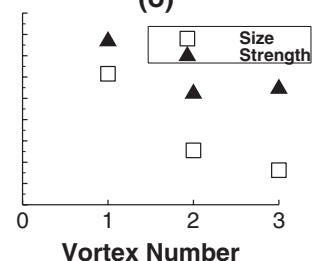

Figure 2. Wake structure at $k=1.82$. (a-c) Vorticity contours. (d-f) Velocity vectors (only alternate velocity vectors are presented). (g-i) streamwise velocity contour. (j-l) lateral velocity contour. $(\mathbf{m}-\mathbf{0})$ size and strength of vortices.

undisturbed position; both the streamwise $(X)$ and cross-stream $(Y)$ directions have been normalized by the chord length. The velocity has been normalized by the free stream velocity and vorticity by $\left(U_{o} / c\right)$.

A counter-clockwise vortex (vortex 2 in figure $2 \mathrm{a}$, shown by red color) is formed on the top-half of the airfoil, while a clockwise vortex forms on its bottom half (figures $2 \mathrm{a}-\mathrm{f}$ ). The upward movement $(+\alpha$ direction) produces a clockwise vortex whereas downward movement $(-\alpha$ direction) produces an anticlockwise vortex. Notice from figure $2 b$ that the vorticity generated in the boundary layer is fed/rolls-up into the vortex. The results suggest a reverse Kármán type wake behind the airfoil. The two vortices move downstream as a couple; the vortices 
however disappear a relatively short distance away from the airfoil. The region between the vortices is marked by the presence of large instantaneous velocity. The particularly large magnitude of both streamwise and cross-stream velocity components is noteworthy from figures $2 \mathrm{~g}-1$. The large magnitude of streamwise velocity remains upon time-averaging; whereas the magnitude of cross-stream velocity reduces substantially upon averaging (figure 5 presented later). The same vortex shedding pattern persists over the entire pitching cycle; a few frames are included here to illustrate this fact. The flow structure does not change qualitatively with an increase in pitching reduced frequency to 3.64 .

As the flapping reduced frequency is increased to 5.46, the reverse Kármán vortex street is again observed (figure 3). The vortex-couple is however now deflected upwards at an angle with respect to the free stream flow direction. Notice that the deflection is biased in the same direction
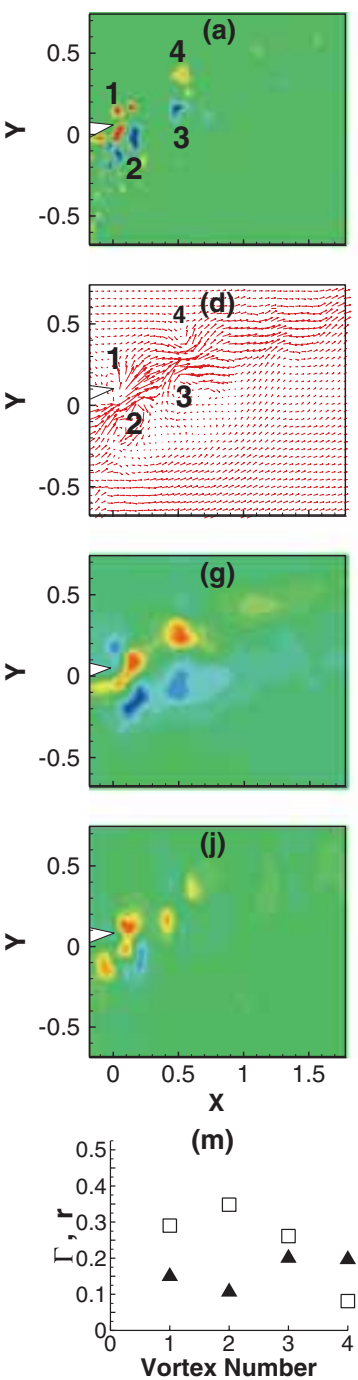
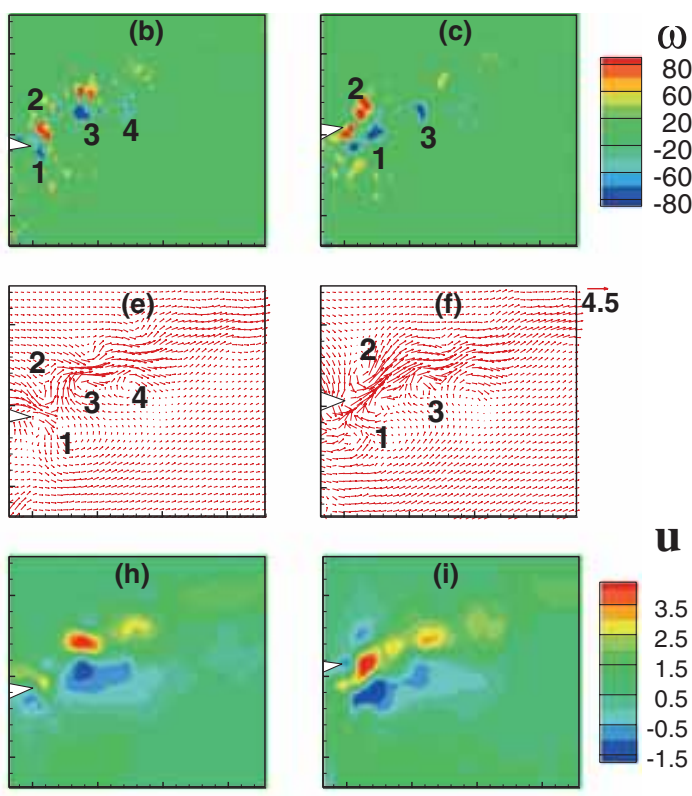

$\mathbf{u}$

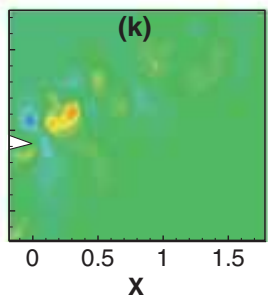

(n)

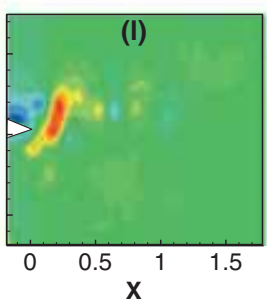

(o)
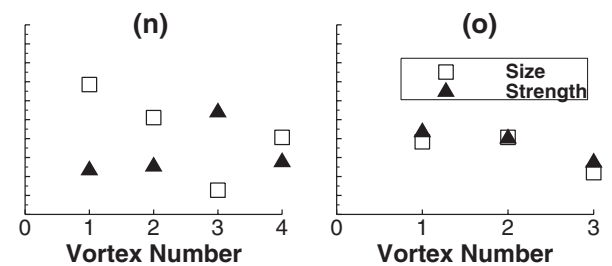

Figure 3. Same as figure 2, but for $k=5.46$. 
irrespective of the position of the airfoil in its pitching cycle, as evident from the subfigures. The drastic reduction in size and strength of the vortices as they move downstream is noteworthy; the reduction in size is up to a factor of three over one chord length distance (figures $3 \mathrm{~m}, 3 \mathrm{n}$ ). As the flapping reduced frequency is further increased to 7.28 the two opposite deflected vortices are still shed into the wake (not shown). The direction and angle of deflection appear to be same for 5.46 and 7.28 of pitching reduced frequency.

The deflection is attributed to physical effects as discussed further in Section 4; and not to any imperfections in the setup or upstream flow conditions. The direction of vortex-couple deflection apparently depends on the initial condition; the effect of initial condition was however not investigated here. Such a deflection has been seen earlier by Jones et al (1998), Heathcote \& Gursul (2007), Godoy-Diana et al (2008, 2009), Cleaver et al (2012), Shinde \& Arakeri (2013) as well. The deflection angle appears to be similar to that seen in Godoy-Diana et al (2008, 2009). The measurements of Heathcote \& Gursul (2007) are however mostly with flexible airfoil in stagnant water. Shinde \& Arakeri (2013) recently noted from their experiments in quiescent water that the deflected jet changed its orientation randomly with time; they termed it as meandering jet.

The direction of deflected vortex shedding changes as the flapping reduced frequency is increased further to 9.10 (figure 4). The angle of deflection appears to be smaller in these cases as compared to those in figure 3. There are instances where the vortex-couples move downward or parallel to the free-stream flow. This reinforces the idea that the deflection is actually due to physical effects and that the direction of vortex shedding depends on the initial condition. The magnitude of jet velocity first increases with frequency and then reduces; this is reflected in the change in the reference vector size (figures $2 \mathrm{f}, 3 \mathrm{f}, 4 \mathrm{f}$ ). The wake structure does not change qualitatively by increasing the reduced frequency from 9.10 to 10.92 .

A few vortices were followed as they advect with the flow, and their size and strength were plotted in figures 2-4(section $\mathrm{m}-\mathrm{O}$ ). Using procedure similar to Kumar et al (2008), the vortex size $\left(r_{c}\right)$ was first determined as the region containing $20 \%$ or more of the vorticity at the vortex center; vortex center is the point with the maximum local vorticity. The vortex strength $(\Gamma)$ is defined at the vorticity contained within the vortex times the vortex area. The vortex for which the data is plotted can be identified by its number in the corresponding subfigure at the top. The vortex strength has been normalized by $\left(U_{o} c\right)$ and the vortex-size by chord length. Interestingly, both the vortex-strength and vortex-size shed from the airfoil are relatively insensitive to change in frequency, over the parameter range covered in the experiments. The reason for the observations noted above is presented in Section 4.

\subsection{Time-averaged flow behavior}

The mean and r.m.s. of streamwise and cross-stream velocity profiles are plotted as a function of the lateral coordinate for a few cases, at three locations downstream of the pitching airfoil. The velocities are normalized by the free-stream velocity and distance by the chord length.

The presence of a reverse Kármán vortex street is accompanied by a jet-like profile (i.e., with momentum excess), as evident from figure 5a. The flow velocity away from the "jet" is slightly less than the free-stream velocity. The lateral coordinate of the maximum velocity for the deflected jet case is clearly different than an un-deflected jet and changes further as the jet proceeds downstream (compare figure 6a against figure 5a). This underscores the fact that the jet stays deflected along the same direction with time. The magnitude of maximum velocity increases with frequency other than at the highest frequency (figure not shown). Further, the magnitude of maximum velocity decreases while the width increases, as the jet proceeds downstream; a typical characteristic of jet flows. 

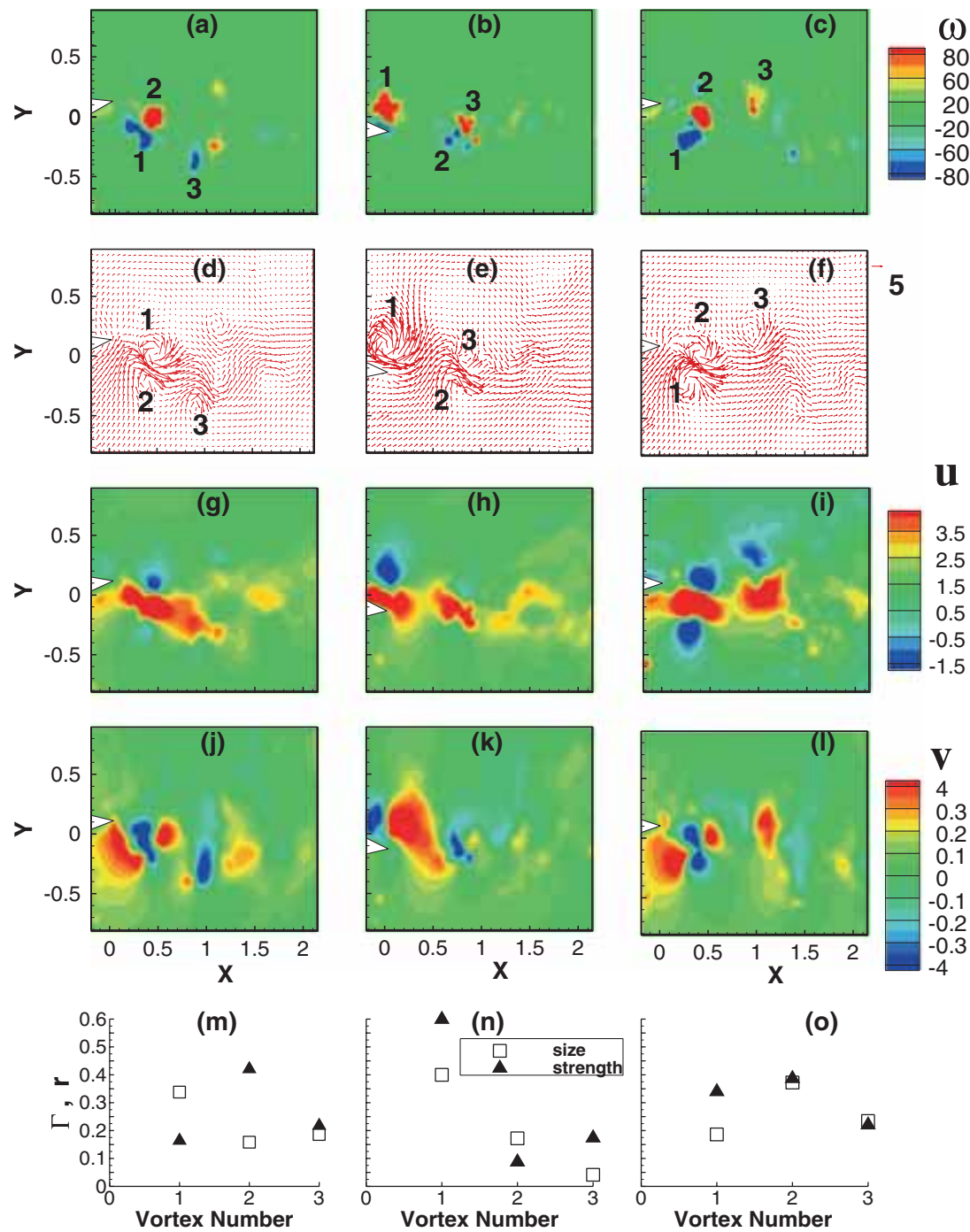

Figure 4. Same as figure 2, but for $k=9.10$.

A mostly positive cross-stream velocity (with only a small region of negative velocity) is noted from figure $5 \mathrm{~b}$. This is probably due to the limited size of the control volume employed in the measurements. The relatively large magnitude of cross-stream velocity as compared to the streamwise velocity (about $25 \%$ of the maximum streamwise velocity) is another noticeable feature revealed by both figures $5 \mathrm{~b}, 6 \mathrm{~b}$. The large magnitude of cross-stream velocity points to strong lateral entrainment of the ambient fluid owing to jet formation and deflection of the jet. (For comparison, the cross-stream velocity for turbulent planar jet in the self-similar region is just 4\% of the maximum streamwise velocity; Agrawal and Prasad 2003).

The normalized streamwise r.m.s. profiles exhibit two local maxima for $k=1.82$ (figure 5c) and three local maxima for $k=7.28$ (figure $6 \mathrm{c}$ ). The maxima correspond to the location of the two vortices. The maximum value of $u_{\mathrm{rms}}$ decreases and the two peaks merge with an increase in the streamwise coordinate. The normalized cross-stream r.m.s. profiles exhibit a single maxima 

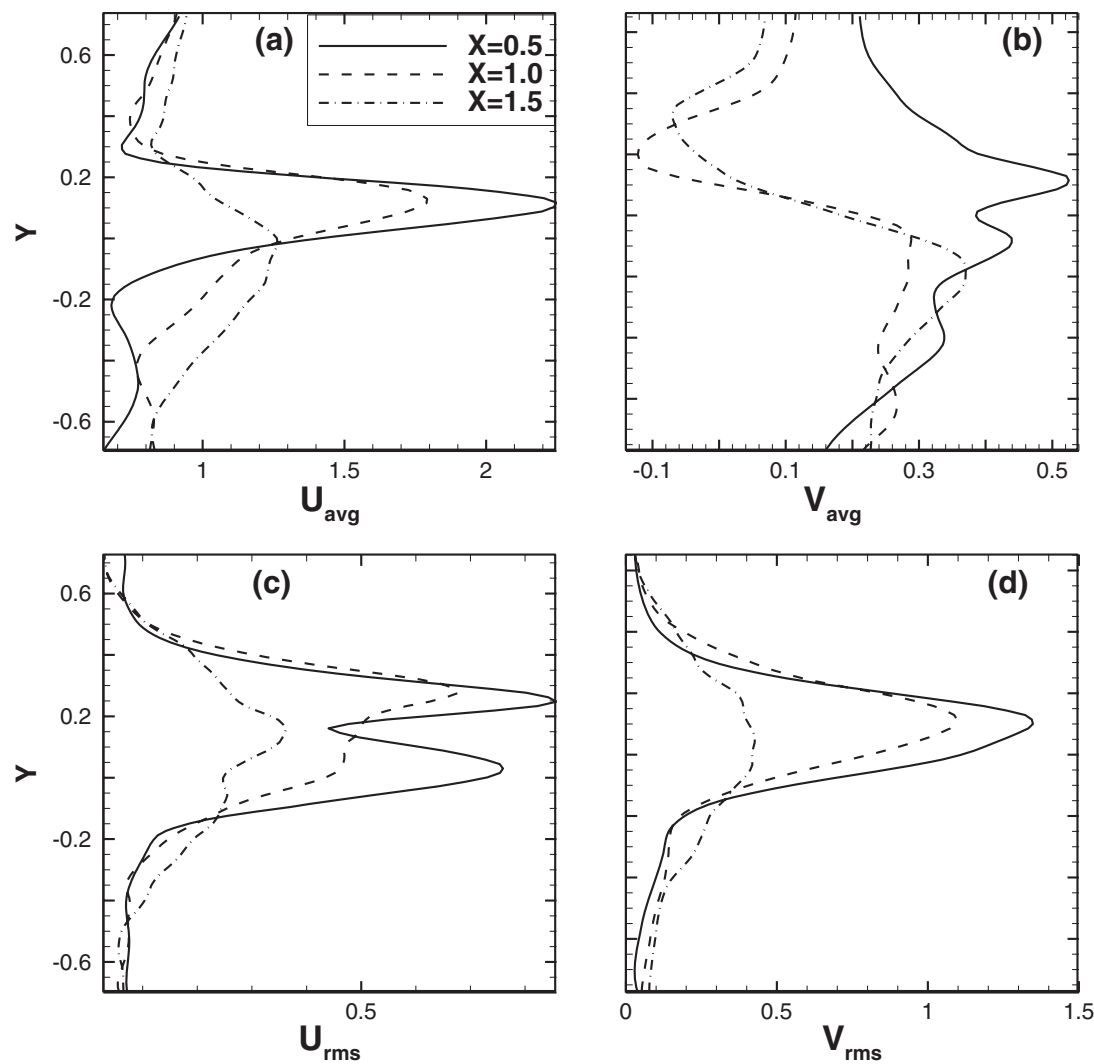

Figure 5. Mean and r.m.s. velocity profiles for three different streamwise stations, at $k=1.82$. (a) Mean streamwise velocity, (b) mean cross-stream velocity, (c) r.m.s. streamwise velocity, (d) r.m.s. cross-stream velocity.

for $k=1.82$ (figure 5d) and two local maxima for $k=7.28$ (figure 6d). Interestingly, the maximum value of $v_{\mathrm{rms}}$ is more than the maximum value of $u_{\mathrm{rms}}$ for both the frequencies. The relatively large value of $u_{\mathrm{rms}}$ is owing to the large change in streamwise velocity as vortices pass through the point. The relatively large value of $v_{\text {rms }}$ is also due to the movement of the vortex; in addition, it points towards substantial lateral movement of the vortex. Such lateral oscillation in the vortex center, as it moves downstream is also evident from the data of Bohl \& Koochesfahani (2009).

The average streamwise velocity data suggests that the amount of excess momentum increases with reduced frequency till 9.10 , but starts to decrease beyond this frequency. The mass flux of the jet first increases and then decreases with frequency, which leads to the observed variation in momentum. This excess momentum can lead to a net production of thrust, as summarized in figure 7. The procedure suggested by Bohl \& Koochesfahani (2009) has been employed in the calculation of thrust coefficient $\left(C_{T}\right)$ :

$C_{T}=\frac{2}{C} \int_{-y_{\min }}^{+y_{\max }}\left\{\frac{U_{a v g}}{U_{0}}\left(\frac{U_{a v g}}{U_{0}}-1\right)+\varepsilon\left(\frac{U_{a v g}}{U_{0}}-1\right)+\left(\frac{u_{r m s}}{U_{0}}\right)^{2}-\left(\frac{v_{r m s}}{U_{0}}\right)^{2}+\frac{1}{2}\left(1-\frac{U_{T}^{2}}{U_{0}^{2}}\right)\right\} d y$, 

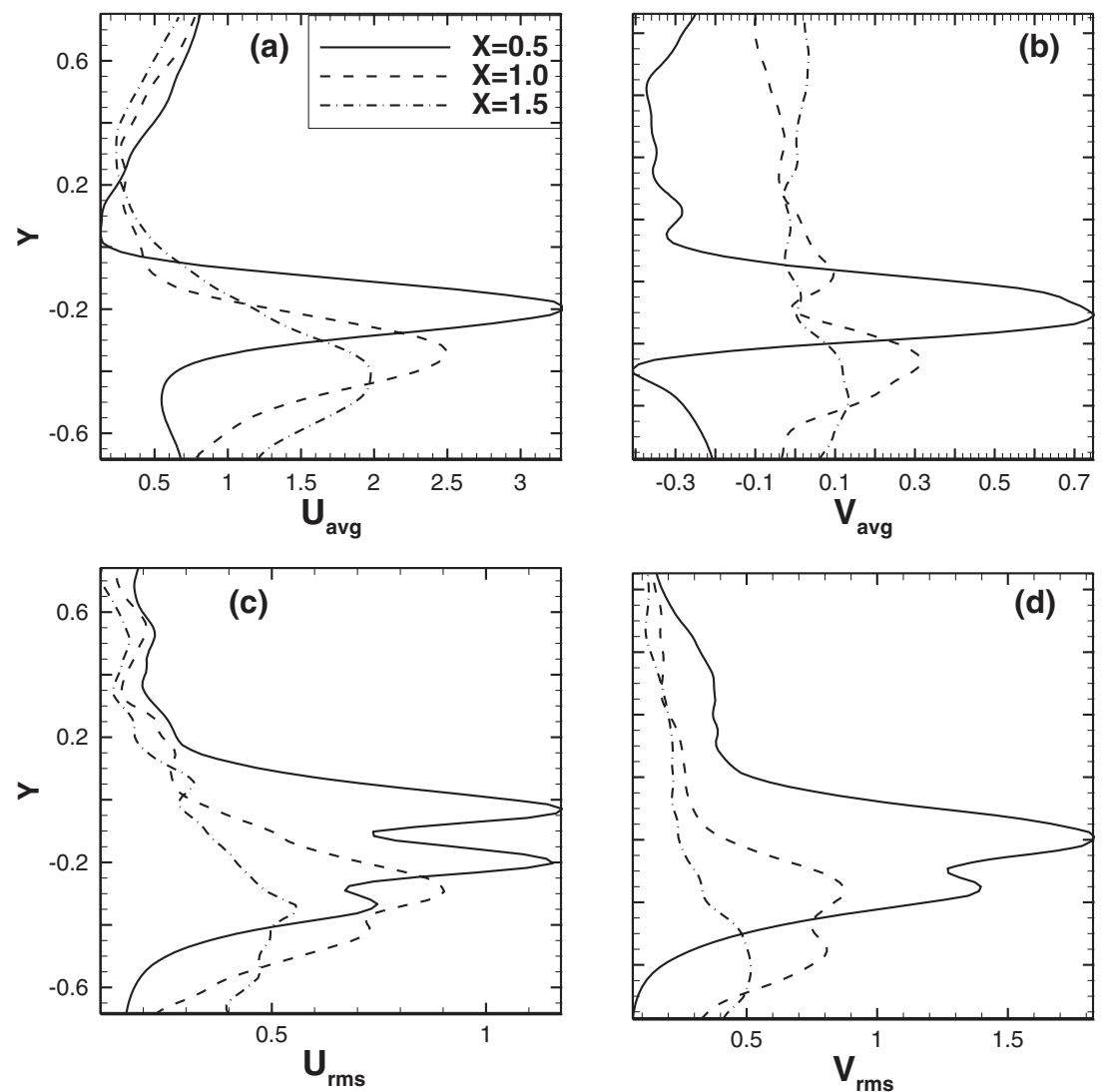

Figure 6. Same as figure 5, but for $k=7.28$.

where $U_{\text {avg }}$ is the average streamwise velocity. Note that the calculation of thrust accounts for the relatively small size of the control volume so that momentum can enter/exit from the sides; this is ensured through the second term in the above equation where $\varepsilon\left(=\left(U_{T}-U_{0}\right) / 2 U_{0}\right)$ accounts for the small variation in lateral velocity $\left(U_{T}\right)$ from the free-stream velocity. Similarly, the change in pressure is estimated through the last three terms. These terms have been obtained from the lateral momentum equation and Bernoulli's equation, as explained in Bohl \& Koochesfahani (2009).

As compared to Yu et al (2013a, b) simulation for pure pitching, the reduced frequency is increased almost two times and Reynolds number by 2.5 times in this work. The drag has increased by 3.36 times as compared to NACA0006 airfoil and by 1.875 times as compared to NACA0030 airfoil. It should also be noted that $C_{T}$ is negative at low frequencies although a reverse Kármán vortex street was observed at these frequencies. This shows that the formation of a reverse Kármán vortex street does not automatically ensure thrust generation; this point was also noted by Bohl \& Koochesfahani (2009). The excess momentum generated by the motion of the airfoil is more than offset by the reduction in pressure on the right surface of the control volume. This pressure difference is brought out by the motion of the airfoil. Note the relatively large value of $C_{T}$ and appearance of maxima. The subsequent increase/decrease in the value of $C_{T}$ is related to an increase/decrease in excess momentum, noted above. 


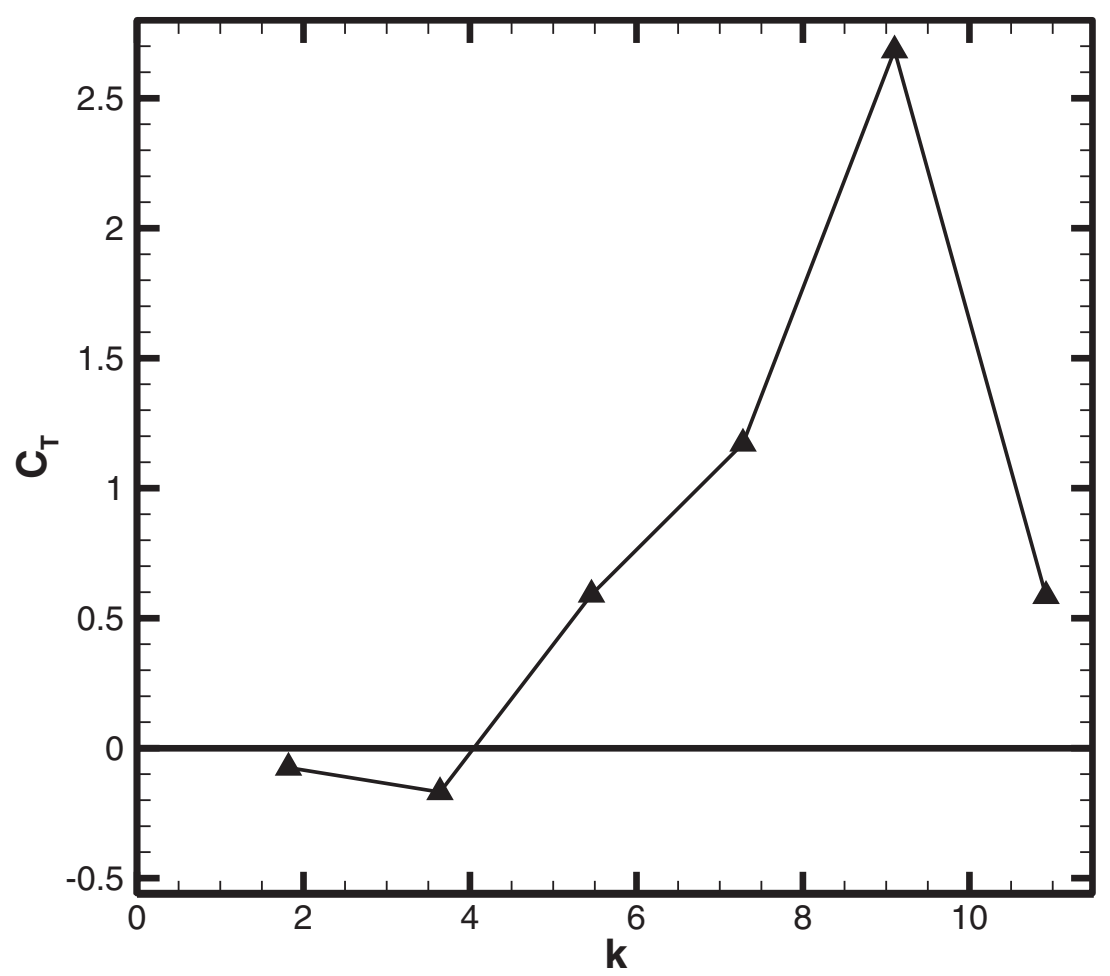

Figure 7. Variation of thrust coefficient with reduced frequency.

\section{Discussion}

We find evidence for a marked reduction in vorticity as the vortices travel downstream. In fact, in several cases, the vorticity becomes so small just after two chord lengths downstream that identification of vortices becomes difficult. This observation is in agreement with that of Bohl \& Koochesfahani (2009). Bohl \& Koochesfahani attributed this large decrease in vorticity to both vorticity diffusion and vortex stretching, with a relative contribution of $70 \%$ and $30 \%$ respectively for their case. They underscored that although the magnitude of streamwise velocity gradient was very small, its contribution in the reduction in vorticity was still appreciable.

There is clear evidence for deflection of vortex street as also noted by Godoy-Diana et al (2008, 2009); such deflected vortex street where however not reported by Schnipper et al (2009) and Bohl \& Koochesfahani (2009). As noted above, the wake deflection appears at an intermediate range of frequency, which is in qualitative agreement with the result of GodoyDiana et al (2009). Those authors further showed that the deflection angle again increases abruptly and rapidly upon increasing the frequency. They attributed this deflection to the formation of a vortex dipole. The vortex dipole entrains more fluid from one of the two sides, leading to a deflection of the flow. This asymmetry in entraining fluid is accentuated by the motion of the airfoil. The direction of deflection is dictated by the motion of the first vortex-dipole; the subsequent vortex dipoles simply follow the direction of the first vortex-dipole.

Schnipper et al (2009) found the occurrence of several different vortex shedding patterns; these vortex patterns are: $2 \mathrm{~S}, 2 \mathrm{P}, 2 \mathrm{P}+2 \mathrm{~S}, 4 \mathrm{P}, 4 \mathrm{P}+2 \mathrm{~S}, 6 \mathrm{P}+2 \mathrm{~S}$ and $8 \mathrm{P}(\mathrm{S}$ stands for single vortex 
and $\mathrm{P}$ stands for vortex pair) shed over a single pitching cycle. They therefore found that up to 16 vortices can be shed in a single pitching cycle. We however find only inverted Kármán vortex street in the parameter range covered in this study. Although the Reynolds number, and pitching frequency and amplitude for the two studies are comparable, there are differences in the airfoil shape employed in the two studies. The airfoil of Schnipper et al (2009) is not from the NACA series, and we suspect that the slightly "bluff" shape of their pitching body yields such a large number of vortices upon pitching.

The magnitude of maximum vorticity and vortex size appear almost invariant of the frequency. Schnipper et al (2009) suggested that the vortices can either form in the boundary layer or at the tip of the airfoil. Keeping other parameters unchanged, the strength of the vortices thus formed scales inversely and directly with the pitching frequency, respectively. Since in the present case, there is an excess momentum, the vortex at the tip of the airfoil is expected to dominate the vortex formed in the boundary layer, and the vortex strength should increase linearly with pitching frequency. A careful examination of vortex formation supports that formation of tip vortices happens in our case; however, we are unable to explain the constancy in vortex strength with frequency.

The present results therefore reveal certain differences with respect to the earlier studies. There is no reason to believe that the earlier results are less (or more) reliable than the present results; more so when the flow features in each of these cases can be both visually and quantitatively verified. We attribute these differences to the differences in the geometry and parameter range employed in the different studies; further studies are needed to reconcile the differences. Our study however points to the important role that airfoil geometry can play in fixing the flow characteristics in such situations. As is evident from several studies, the flow features and magnitude of forces vary with time in a flexible airfoil. This is clearly due to change in the airfoil geometry with time along with other dynamic effects. Here, we argue that airfoil geometry plays an important role even with rigid airfoils.

\section{Concluding remarks}

An experimental study of a pitching airfoil in the presence of a free-stream flow is undertaken in this work. The focus is on the low Reynolds number regime which has been inadequately studied in the literature. Detailed vortex-structure and velocity distribution are presented as a function of pitching frequency.

We are able to confirm some results from the literature such as deflection of jet at certain pitching frequencies and large reduction in vortex strength with streamwise distance. Our results however indicate some differences with respect to reported results - absence of formation of more than two vortices per pitching cycle; constancy of vortex strength with respect to pitching frequency; existence of a maximum in coefficient of thrust at a reduced frequency of 9.10. We argue that these differences in results may be because of difference in the airfoil geometry. Further work is required to better understand the effect of airfoil geometry on the ensuing wake structure and thrust generation. The results therefore elucidate the flow around a pitching airfoil and can be useful for benchmarking future numerical studies.

\section{References}

Agrawal A and Prasad A K 2003 Integral solution for the mean flow profiles of turbulent jets, plumes and wakes. J. Fluid Eng. 125(5): 813-822 
Bohl D G and Koochesfahani M M 2009 MTV measurements of the vortical field in the wake of an airfoil oscillating at high reduced frequency. J. Fluid Mech. 620: 63-88

Buchholz H J J and Smits A J 2008 The wake structure and thrust performance of a rigid low-aspect ratio pitching panel. J. Fluid Mech. 603: 331-365

Cleaver D J, Wang Z and Gursul I 2012 Bifurcating flows of plunging aerofoils at high Strouhal numbers. J. Fluid Mech. 708: 349-376

Chandar D D J and Damodaran M 2010 Computation of unsteady low Reynolds number free-flight aerodynamics of flapping wings. J. Aircraft 47(1): 141-150

Godoy-Diana R, Aider J L and Wesfreid J E 2008 Transitions in the wake of a flapping foil. Phys. Rev. E 77: 016308

Godoy-Diana R, Marais C, Aider J L and Wesfreid J E 2009 A model for the symmetry breaking of the reverse Benard-von Karman vortex street produced by a flapping foil. J. Fluid Mech. 622: 23-32

Freymuth P 1988 Propulsive vortical signature of plunging and pitching airfoils. AIAA J. 26: 881-883

Govardhan R N and Arakeri J H 2011 Fluid mechanics of aquatic locomotion at large Reynolds numbers. $J$. Indian Inst. Sci. 91: 3

Green M A and Smits A J 2008 Effects of three-dimensionality on thrust production by a pitching panel. $J$. Fluid Mech. 615: 211-220

Heathcote $\mathrm{S}$ and Gursul I 2007 Jet switching phenomenon for a periodically plunging airfoil. Phys. Fluids 19: 027104

Jones K D, Dohring C M and Platzer M F 1998 Experimental and computational investigation of the Knoller-Betz effect. AIAA J. 36(7): 1240-1246

Koochesfahani M M 1989 Vortical patterns in the wake of an oscillating airfoil. AIAA J. 27(9): 1200-1205

Kumar S R, Sharma A and Agrawal A 2008 Simulation of flow around a row of square cylinders. J. Fluid Mech. 60: 369-392

Platzer M F, Jones K D, Young J and Lai J C S 2008 Flapping-wing aerodynamics: Progress and challenges. AIAA J. 46(9): 2136-2149

Ramamurti R and Sandberg W 2001 Simulation of flow about flapping airfoils using finite element incompressible flow solver. AIAA J. 39(2): 253-260

Sarkar S and Venkatraman K 2006 Numerical simulation of thrust generating flow past a pitching airfoil. Comput. Fluids 35: 16-42

Schnipper T, Andersen A and Bohr T 2009 Vortex wakes of a flapping foil. J. Fluid Mech. 633: 411-423

Sewatkar C M, Patel R, Sharma A and Agrawal A 2012 Flow around six in-line square cylinders. J. Fluid Mech. 710: 195-233

Shinde S Y and Arakeri J H 2013 Jet meandering by a foil pitching in quiescent fluid. Phys. Fluids 25(4): 041701

Shyy W, Aono H, Chimakurthi S K, Trizila P, Kang C-K, Cesnik C E S and Liu H 2010 Recent progress in flapping wing aerodynamics and aeroelasticity. Prog. Aerospace Sci. 46: 284-327

Yu M, Wang Z J and Hu H 2013a High Fidelity numerical simulation of airfoil thickness and kinematics effects on flapping frequency. J. Fluids Struct. 42: 166-186

Yu M, Wang Z J and Hu H 2013b High fidelity numerical simulation of airfoil thickness and kinematics effects on flapping airfoil propulsion. J. Fluids Struct. 42: 166-186 